\title{
Article
}

\section{Worm-like micelles of triblock copolymer of ethylene oxide and styrene oxide characterised using light scattering and Taylor dispersion analysis}

Zhou, Zhengyuan, Chhabria, Vikesh Nandkishore, D'Emanuele, Antony and Forbes, Robert Thomas

Available at http://clok.uclan.ac.uk/34578/

Zhou, Zhengyuan ORCID: 0000-0002-6804-3897, Chhabria, Vikesh Nandkishore, D'Emanuele, Antony and Forbes, Robert Thomas ORCID: 00000003-3521-4386 (2020) Worm-like micelles of triblock copolymer of ethylene oxide and styrene oxide characterised using light scattering and Taylor dispersion analysis. International Journal of Pharmaceutics, 588 . ISSN 03785173

It is advisable to refer to the publisher's version if you intend to cite from the work. http://dx.doi.org/10.1016/j.j.jpharm.2020.119758

For more information about UCLan's research in this area go to http://www.uclan.ac.uk/researchgroups/ and search for <name of research Group>.

For information about Research generally at UCLan please go to http://www.uclan.ac.uk/research/

All outputs in CLoK are protected by Intellectual Property Rights law, including Copyright law. Copyright, IPR and Moral Rights for the works on this site are retained by the individual authors and/or other copyright owners. Terms and conditions for use of this material are defined in the policies page. 
7 a School of Pharmacy and Biomedical Sciences, University of Central Lancashire, Preston PR1 2HE,

$8 \quad U K$

9 b Leicester School of Pharmacy, De Montfort University, The Gateway, Leicester LE1 9BH, UK

$11 *$ To whom correspondence should be addressed: Zhengyuan Zhou zzhou2@uclan.ac.uk

\section{Abstract}

A triblock ESE copolymer $\left(\mathrm{E}_{16} \mathrm{~S}_{8} \mathrm{E}_{16}, \mathrm{~S}=\right.$ styrene oxide and $\mathrm{E}=$ ethylene oxide $)$ was synthesised by sequential oxyanionic copolymerisation of styrene oxide followed by ethylene oxide. Light scattering

17 studies demonstrated a shape transition from spherical micelles to worm-like micelles above a critical temperature of approximately $18^{\circ} \mathrm{C}$. Taylor dispersion analysis (TDA) also indicated a size growth when the temperature increased from 25 to $40{ }^{\circ} \mathrm{C}$ due to the formation of worm-like micelles. The hydrodynamic radii and diffusion coefficients obtained by these two techniques were in good agreement. The solubility of a hydrophobic drug, terfenadine, in dilute micellar solutions of the copolymer was increased at least 20 -fold under the conditions. The transition to worm-like micelles at raised temperatures led to enhanced solubilisation capacities due to a larger hydrophobic core volume. 
24 The behaviour of the novel ESE copolymer shows the utility of TDA to follow conformational changes using nanolitre quantities and explore critical quality attributes for this type of drug delivery system.

\section{Key words:}

Poly(oxyalkylenes), Worm-like micelles, Dynamic light scattering, Taylor dispersion analysis, Drug solubilisation.

\section{Introduction}

The use of amphiphilic block copolymers for drug solubilisation and delivery has been extensively investigated, as reviewed in a number of publications over the last decade (Torchilin, 2001; Adams et al., 2003; Chiappetta and Sosnik, 2007). Nonionic amphiphilic block copolymers are considered to be more suitable for use in drug solubilisation and drug delivery systems since they have lower toxicity and greater biological compatibility than cationic and anionic surfactants (Grindel et al., 2002). Their low critical micelle concentration results in a high degree of micellization, and highly stable micelles can be formed at comparatively low concentrations. The micelle core, which is composed of hydrophobic components, provides a suitable microenvironment for the incorporation of lipophilic drugs, while the hydrophilic micelle corona serves as a stabilising interface between the hydrophobic core and the external medium.

Block copoly(oxyalkylene)s, consisting of a hydrophilic poly(ethylene oxide) (E) block and a hydrophobic block, e.g. poly(propylene oxide) (P), poly(1, 2-butylene oxide) (B) or poly(styrene oxide) 
micellisation of block copoly(oxyalkylene)s with various architectures have been widely investigated

50 (Booth et al., 2006). Significant solubility enhancement for poorly water-soluble drugs can be achieved

51 in dilute micellar solutions of block copoly(oxyalkylene)s at ambient temperatures. (Crothers et al.,

52 2005; Attwood et al., 2007; Zhou et al., 2008). It is understood that solubilisation capacity is dependent

53 on the hydrophobicity of core-forming blocks and the volume of the hydrophobic cores. For block

54 copoly(alkylene)s with long block lengths, which normally form spherical micelles, the volume of

55 micellar cores is limited by the stretched length of hydrophobic blocks. However, Booth and Attwood

56 have indicated that copolymers with short E blocks, (which leads to high association numbers), and

57 with short hydrophobic blocks, (which places a low ceiling on the radius of a spherical micelle), are

58 more likely to form elongated micelles (Booth and Attwood, 2000). A range of diblock

copoly(oxyalkylene)s, e.g. $\mathrm{E}_{17} \mathrm{~B}_{12}$ (Chaibundit et al., 2005), $\mathrm{E}_{13} \mathrm{~B}_{10}$ (Zhou et al., 2008), $\mathrm{E}_{11} \mathrm{~B}_{8}$

60

(Chaibundit et al., 2002) and $\mathrm{E}_{17} \mathrm{~S}_{8}$ (Yang et al., 2003), have been synthesised and investigated for their

micelle properties using light scattering. An abrupt increase of hydrodynamic radii and aggregation

number with temperature was observed for these copolymers, which indicates the formation of worm-

like micelles. Solubilisation studies show that these worm-like copolymer solutions have much greater

solubilisation capacities than those of conventional spherical micelles under similar conditions.

Triblock copolymers, e.g. $\mathrm{E}_{20} \mathrm{~S}_{10} \mathrm{E}_{20}$, with relatively short block length, are also assumed to be able to

form elongated micelles, and as previously reported that their solubilisation capacity for griseofulvin is

much higher than small spherical micelles (Crothers et al., 2005). However, a full characterisation of

micelle properties has never been performed for such triblock ESE copolymers in order to understand

their micellisation behaviour. For triblock copolymers to be useful for drug delivery applications such

characterisations are essential to evaluate critical quality attributes.

In this work, we aim to prepare a triblock ESE copolymer with well-chosen block lengths that can 
determined using light scattering and Taylor dispersion analysis (TDA) techniques. TDA is a technique for the determination of diffusion coefficients and hydrodynamic radii, presented by Geoffrey Taylor in 1953 (Taylor, 1953) and further developed by Aris in 1956 (Aris, 1956). The principal of this technique is based on band broadening of a solute plug in a straight capillary under laminar flow conditions. TDA has been explored for the size measurement of many substances, e.g. protein aggregates (Hawe et al., 2011), cyclodextrin-drug aggregates (Zaman et al., 2017), super-paramagnetic nanoparticles (Lemal et al., 2018), poly-L-lysine dendrigrafts (Cottet et al., 2007), and micelles and microemulsions (Chamieh et al., 2015). Here TDA is first employed to investigate the shape transition of micelles of block copolymers upon changes in temperature. The specific objectives of this work are to synthesise and characterise a triblock ESE copolymer, investigate the micellisation behaviour of the copolymer using light scattering and Taylor dispersion analysis techniques, and evaluate the solubilisation capacities of the copolymer for a poorly water-soluble model drug utilising a UV assay.

\section{Experimental}

\section{Materials}

Ethylene oxide, styrene oxide, terfenadine, Pluronic F127 were purchased from Sigma-Aldrich (UK). HPLC grade THF and methanol were obtained from Fisher Scientific Ltd. UK. NMR grade chloroform-d and methanol-d were from Cambridge Isotope Laboratories (USA).

\section{3}

\section{ESE block copolymers}

The copolymer was prepared by sequential oxyanionic copolymerisation of styrene oxide followed by ethylene oxide (Fig. S1). The general method has been described in detail previously (Yang et al., 2003b). Briefly, the difunctional initiator was potassium hydroxide and water. Freshly dried styrene 
oxide was transferred into the ampoule and heated at $85^{\circ} \mathrm{C}$ for 8 weeks. Then ethylene oxide was

100 distilled into the ampoule and kept at $65^{\circ} \mathrm{C}$ for about 3 weeks until polymerisation was completed. The 101 copolymer was characterised by gel permeation chromatography (GPC, Agilent 1260 Infinity with

102 triple detectors and two Agilent PLgel Mixed-D columns, tetrahydrofuran eluent, calibrated with

103 poly(styrene) standards for measurement of molecular weight and polydispersity. ${ }^{1} \mathrm{H}$ and ${ }^{13} \mathrm{C}$ NMR

104 spectroscopies (Bruker Avance 400, Bruker, Coventry, UK) were used to determine the composition of 105 the copolymer (Figs. S2 and S3). The assignment for the peaks of ESE copolymers was made 106 according to relevant references (Heatley et al., 1991).

107

108

\section{Critical micelle concentration}

The critical micelle concentration (CMC) of the ESE copolymer at $20{ }^{\circ} \mathrm{C}$ was determined by surface tension measurement using the pendant drop method. An FTA1000 video system (First Ten Ångstroms Inc) was used to visualise liquid drops formed on the tip of a stainless-steel needle (20 gauge). The image was taken using aperture 22 with $50 \%$ brightness and contrast. Surface tension of aqueous polymer solutions with a range of concentration from 0.001 to $2 \% \mathrm{w} / \mathrm{v}$ was calculated via drop-shape analysis. Ten measurements were recorded for each sample and the results averaged. The standard deviation of the drop-shape analysis was approximately $\pm 0.5 \mathrm{mN} \mathrm{m}^{-1}$ and the measurement error was less than $5 \%$.

\section{Light scattering}

The micelle properties of the copolymer were measured by static and dynamic light-scattering techniques. Solutions were filtered through Millipore Millex filters ( $0.22 \mu \mathrm{m}$ porosity) into glass scattering cuvettes. Static light scattering (SLS) intensities were measured at temperatures in the range 
122 of $15-40^{\circ} \mathrm{C}$ using Malvern Zetasizer Nano ZS. The intensity scale was calibrated against scattering

123 from toluene. Analysis of the SLS results was based on the Debye equation,

124

125

126

127

128

129

131

$$
K^{*} c / R_{\theta}=1 / M_{\mathrm{W}}+2 A_{2} c \ldots
$$

where $R_{\theta}$ is the ratio of scattered light to incident light of the sample, $c$ is the concentration (in $\mathrm{g} \mathrm{dm}^{-3}$ ), $M_{w}$ is the weight-average molar mass of the solute, $A_{2}$ is the second virial coefficient (higher coefficients being neglected), and $K^{*}$ is the appropriate optical constant, including the specific refractive index increment, $\mathrm{d} n / \mathrm{d} c$. Values of $\mathrm{d} n / \mathrm{d} c$ were measured using a refractometer (RM50, Mettler Toledo). The data were in good agreement with the equation established previously for a range of block copolymers of ethylene oxide and styrene oxide (Yang et al., 2003b). Values of the weightaverage molar mass of the micelles $\left(M_{\mathrm{w}, \mathrm{mic}}\right)$ were obtained from Debye plots by extrapolation to zero concentration.

Dynamic light scattering (DLS) were measured with the same instrument at a range of temperatures. The correlation functions were analysed to determine intensity fraction distributions of the apparent diffusion coefficient $\left(D_{\mathrm{app}}\right)$ and the apparent hydrodynamic radius $\left(r_{\mathrm{h}, \mathrm{app}}\right)$ via the Stokes-Einstein equation.

where $k$ is the Boltzmann constant and $\eta$ is the viscosity of water at temperature $T$.

$$
r_{\mathrm{h}, \mathrm{app}}=k T /\left(6 \pi \eta D_{\mathrm{app}}\right)
$$


147 hydrodynamic radii and diffusion coefficients of ESE copolymer in solution. A sample solution is

148 injected into the running buffer solution driven by a pressure pump into the fused silica capillary. The

149 solute plug is imaged at two windows by a UV detector. The instrument calculates band broadening

150 from the absorbance recorded at a given wavelength versus time.

151 The Viscosizer 200 was used with a fused silica capillary of $75 \mu \mathrm{m}$ internal diameter and a total

152 length of $130 \mathrm{~cm}$. The length to window 1 is $45 \mathrm{~cm}$ and to window 2 is $85 \mathrm{~cm}$, respectively.

153 Measurement were carried out using an optical filter at $214 \mathrm{~nm}$. The instrument was calibrated by stray

154 light corrections using an appropriate stray light test solution of $10 \mathrm{mg} / \mathrm{ml}$ L-tryptophan in water that

155 shows strong UV absorption. This minimises the effect of stray light (originated from the spectral

156 components of the instrument) on the image element of the system and allows accurate cross talk

157 between two measuring windows. The hydrodynamic radius of $1 \% \mathrm{w} / \mathrm{v}$ ESE solution was measured at

158 various temperatures. A dilute ESE solution above CMC $(0.1 \% \mathrm{w} / \mathrm{v})$ was used as the running buffer to

159 avoid possible morphology change during diffusion. The sample solutions were incubated for an hour

160 at the experimental temperatures $\left(25^{\circ} \mathrm{C}\right.$ and $\left.40^{\circ} \mathrm{C}\right)$ prior to loading. The samples were run in triplicate

161 as the sequence followed: rinse and refill running buffer at 2000 mbar for 2 mins, reset baseline for

$1621 \mathrm{~min}$ and load sample for $20 \mathrm{sec}$ at $140 \mathrm{mbar}$, and run the test at $140 \mathrm{mbar}$. The software automatically

163 processed absorbance versus time data to obtain $r_{\mathrm{h}}$ based on the equation shown below (Hawe et al.,

164 2011):

165

$$
r_{\mathrm{h}}=4 k_{\mathrm{b}} T\left(\tau_{2}^{2}-\tau_{1}^{2}\right) /\left(\pi \eta r^{2}\left(t_{2}-t_{1}\right)\right)
$$

166 Where $k_{\mathrm{b}}$ is the Boltzman constant; $\eta$ is the viscosity; $r$ is the radius of the capillary; $T$ is the

167 temperature; $t_{1}$ and $t_{2}$ correspond to peak centre times at the first and second windows; $\tau_{1}$ and $\tau_{2}$ are the 168 corresponding standard deviations of band broadening. 


\section{Drug solubilisation}

171 The solubilisation capacities of micellar solutions of the ESE copolymer for the model drug

172 terfenadine were measured. The solubilisation in EPE copolymer F127 were also measured under the 173 same conditions to compare the solubility enhancement.

174 The method has been described before (Zhou et al, 2008, 2009). Briefly, saturated drug-loaded 175 solutions were prepared by adding excess drug $(10 \mathrm{mg})$ in $2 \mathrm{ml}$ of 1 and $2 \mathrm{wt} \%$ micellar solutions. The 176 samples were incubated at 25 or $37^{\circ} \mathrm{C}$ for 2 days and then filtered $(0.45 \mu \mathrm{m}$ Millipore $)$ to remove any unsolubilised drug. The drug solubility was determined by UV assay. The filtrate was diluted with methanol and the UV absorbance measured at $230 \mathrm{~nm}$. Calibration with drug alone yielded satisfactory Beer's Law plots (Fig. S4). All measurements were carried out in triplicate and the results averaged.

\section{Results and discussion}

182

\section{ESE copolymer}

The block length and composition of ESE copolymer was determined by NMR with reference to the peak assignments described by Heatley et al. 1991. For ${ }^{13} \mathrm{C}$ NMR, the integrals of the peaks from polymer backbone and end groups were used to determine the block lengths. In ${ }^{1} \mathrm{H}$ NMR, the peaks of aromatic protons were between 7.0-7.6 ppm while all the aliphatic protons peaks were between 3.3 to 4.7 ppm, which provides the information on the relative ratio of $\mathrm{E}$ and $\mathrm{S}$ block lengths. The molecular formula calculated in combination of both spectra was $\mathrm{E}_{16} \mathrm{~S}_{8} \mathrm{E}_{16}\left(\mathrm{MW} 2368 \mathrm{~g} \mathrm{~mol}^{-1}\right.$ ). The GPC measurements revealed the molecular weight of the copolymer to be $2433 \mathrm{~g} \mathrm{~mol}^{-1}$ with a polydispersity of 1.13, which is in good agreement with findings from NMR.

192

193 Critical micelle concentration. 
194 The critical micelle concentration of copolymer $\mathrm{E}_{16} \mathrm{~S}_{8} \mathrm{E}_{16}$ was measured at room temperature

195 (approx. $20^{\circ} \mathrm{C}$ ). Fig. 1 shows the plot of surface tension versus logarithm concentration for the ESE

196 copolymer. The commencement of curvature is an indication of the start of the micellisation process.

197 The CMC determined from the inflection point was $0.73 \mathrm{~g} \mathrm{dm}^{-3}$. The CMCs for a range of ESE

198 copolymers with various block lengths have been reported previously (Yang et al., 2003b). The values

199 of CMC are mainly related to the length of hydrophobic S blocks. Compared to diblock copolymers,

200 the S block of triblock ESE copolymers are more extended due to the two E blocks and thus show

201 higher CMCs. Copolymer $\mathrm{E}_{82} \mathrm{~S}_{8} \mathrm{E}_{82}$, with a comparable $\mathrm{S}$ block length, has a CMC of $0.51 \mathrm{~g} \mathrm{dm}^{-3}$ at 20

$202{ }^{\circ} \mathrm{C}$, which is within the same range as $\mathrm{E}_{16} \mathrm{~S}_{8} \mathrm{E}_{16}$. At higher temperatures, the $\mathrm{CMC}$ values decrease due

203 to a less favourable interaction between water and hydrophilic E blocks. In the micellisation and

204 solubilisation study of this work, the micellar solutions of copolymer $\mathrm{E}_{16} \mathrm{~S}_{8} \mathrm{E}_{16}$ were investigated at $1 \%$

$205 \mathrm{w} / \mathrm{v}$ or above $\left(10 \mathrm{~g} \mathrm{dm}^{-3}\right)$, which is much higher than its CMC. Hence it was assumed that micellisation 206 is complete at the concentration and temperature.

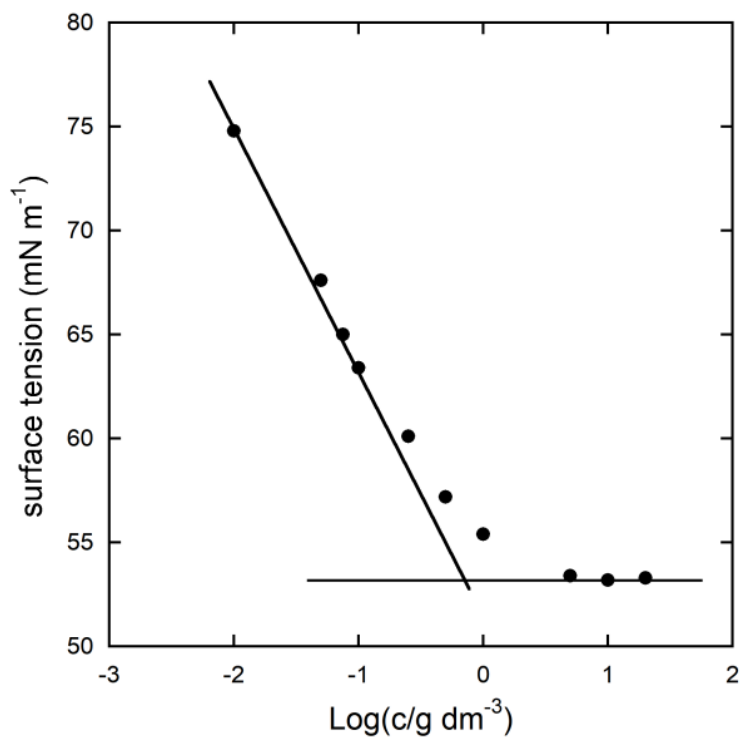


212 Micellisation behaviour of poly(oxyalkylene)s is mainly determined by the hydrophobicity and 213 length of their hydrophobic blocks. Our previous work indicates that the temperature of worm-like 214 micelles formation decreases with an increase of hydrophobic block length (Zhou et al., 2008). In this 215 work we prepared the copolymer $\mathrm{E}_{16} \mathrm{~S}_{8} \mathrm{E}_{16}$ with an $\mathrm{E}: \mathrm{S}$ ratio of $4: 1$ that is anticipated to form worm-like 216 micelles at ambient temperature while possessing good solubility in water. The micelle properties of 217 the ESE copolymer in aqueous solution at different temperatures $\left(<40^{\circ} \mathrm{C}\right)$ were determined using 218 static and dynamic light scattering. Care was taken to work under conditions which ensured optical 219 clarity for the solutions. Dynamic light scattering was performed at different temperatures to obtain 220 intensity fraction distributions of hydrodynamic radii for the ESE copolymer. Figure 2 shows the change in the intensity fraction distribution of $\log \left(r_{\mathrm{h}}\right)$ as the temperature of a $1 \% \mathrm{w} / \mathrm{v}$ solution of $\mathrm{E}_{16} \mathrm{~S}_{8} \mathrm{E}_{16}$ is increased from 5 to $40{ }^{\circ} \mathrm{C}$. The size distribution curves indicate a relatively narrow distribution of small spherical micelles at $5{ }^{\circ} \mathrm{C}$, and a broader distribution of large elongated micelles at $40{ }^{\circ} \mathrm{C}$. The shift in peak position to higher values of $r_{\mathrm{h}}$ indicates a transition from compact to worm-like micelles in solutions of copolymer $\mathrm{E}_{16} \mathrm{~S}_{8} \mathrm{E}_{16}$.

The temperature dependence of $r_{\mathrm{h}}$ of a $1 \% \mathrm{w} / \mathrm{v}$ solution of $\mathrm{E}_{16} \mathrm{~S}_{8} \mathrm{E}_{16}$ is shown in Fig. 3. The $r_{\mathrm{h}}$ almost remains constant at low temperatures. When the temperature increases above a certain value, the curve rises from the baseline and increases gradually, followed by an abrupt increase at high temperatures. Such behaviour indicates that the size of the micelles exceeds the limit for spherical micelles. The commencement of curvature is used as an indication of the transition from spherical to elongated micelles. The transition temperature $\left(\mathrm{ca} .18^{\circ} \mathrm{C}\right)$ is determined more reasonably by the intersection point of the tangent line of the curvature and the baseline. The same phenomenon was also observed for diblock $\mathrm{EB}\left(\mathrm{E}_{17} \mathrm{~B}_{12}, \mathrm{E}_{13} \mathrm{~B}_{10}\right.$ and $\left.\mathrm{E}_{11} \mathrm{~B}_{8}\right)$ (Zhou et al., 2008) and $\mathrm{ES}\left(\mathrm{E}_{17} \mathrm{~S}_{8}\right)$ (Yang et al., 2003) copolymers that have been proven to form worm-like micelles at raised temperature. The increase in 
235 hydrodynamic radius above the transition temperature is consistent with a gradual increase in the size 236 of elongated micelles. The transition temperature is dependent on the hydrophobicity of core-forming 237 blocks and shows a decreasing tendency with increasing hydrophobic block lengths. However, such 238 temperature effect was absent for the copolymers with long E block lengths. For a range of triblock 239 ESE copolymers, e.g. $\mathrm{E}_{82} \mathrm{~S}_{8} \mathrm{E}_{82}$, the weight-average micelle molar mass and the aggregation number 240 remain consistent with increasing temperature (Yang et al., 2003b). Such a finding indicates that the 241 micelle size has almost reached the limit for spherical micelle in this system.

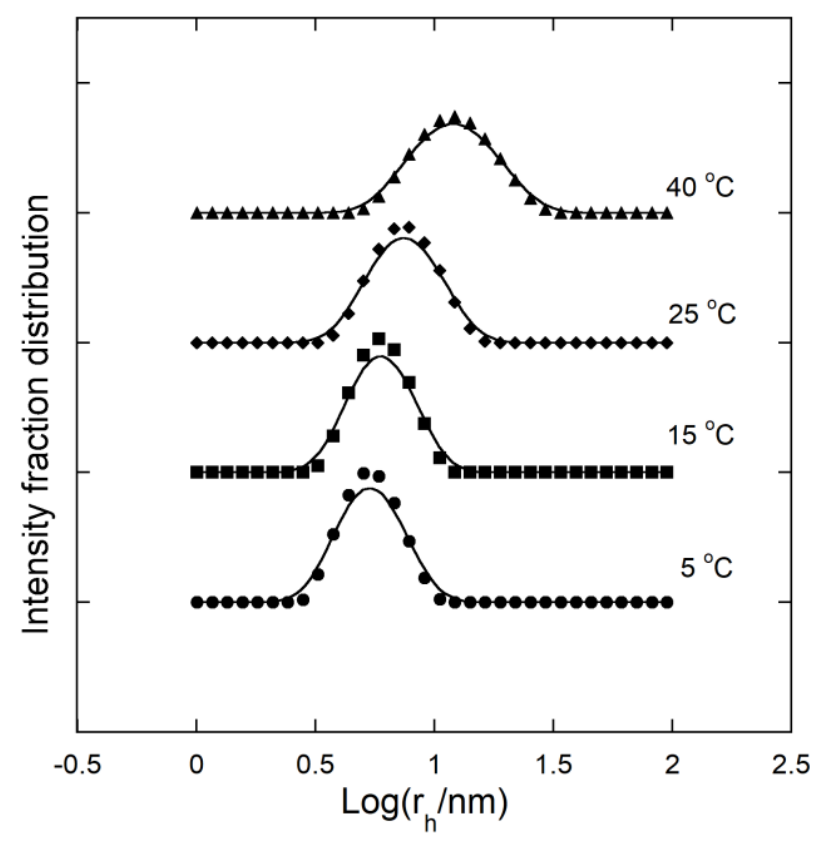

244 Figure 2. Comparison of micelle size distributions of a $10 \mathrm{~g} \mathrm{dm}^{-3}$ solution of copolymer $\mathrm{E}_{16} \mathrm{~S}_{8} \mathrm{E}_{16}$ at the temperatures indicated measured by DLS. 


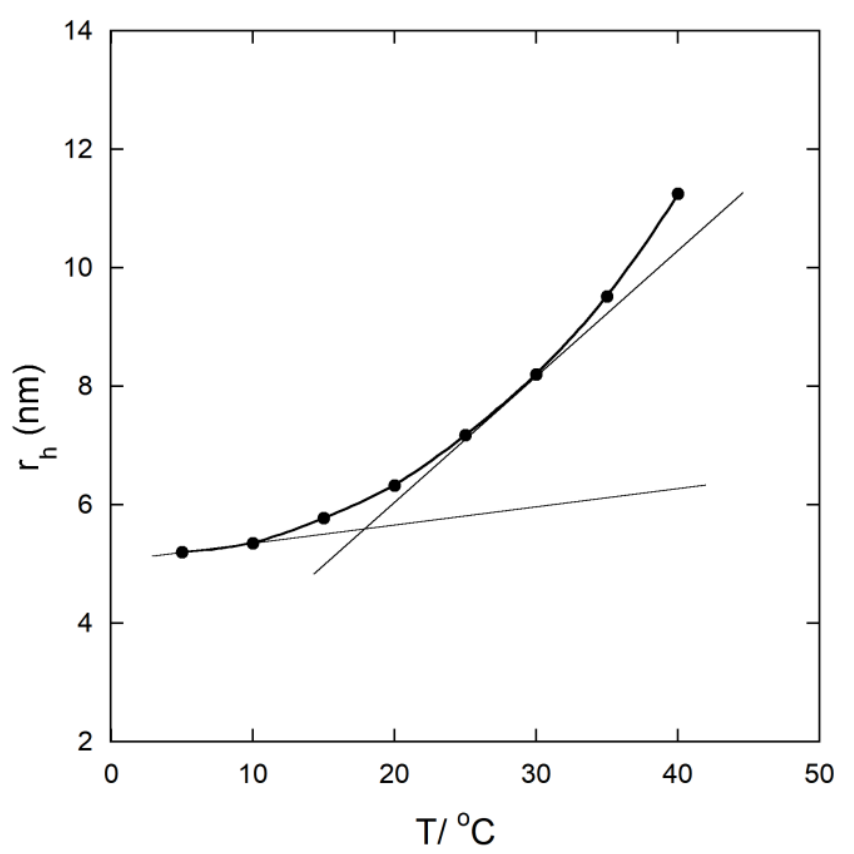

Figure 3. Temperature dependence of hydrodynamic radius of a $10 \mathrm{~g} \mathrm{dm}^{-3}$ solution of copolymer $\mathrm{E}_{16} \mathrm{~S}_{8} \mathrm{E}_{16}$ measured by DLS.

SLS

Debye plots were used to obtain the average molar masses, association numbers and thermodynamic radii of the micelles (Fig. 4). Each data set was fitted with a curve, based on scattering theory for hard spheres using the Carnahan-Starling analysis (Carnaham and Starling, 1969). However, at higher temperatures, more than one species of micelle exists in the micellar solutions due to the transition of spherical micelles to elongated micelles. Hence, the results obtained from the Debye plot were the average values for all the micelles in the solution. The intercept of each Debye plot yields the reciprocal weight-average micelle molar mass $\left(M_{\mathrm{w}, \mathrm{mic}}\right)$ and the curvature gives values for the thermodynamic expansion factor $(\delta t)$. The weight-average association number $\left(N_{\mathrm{w}}\right)$ was subsequently calculated from $M_{\mathrm{w}, \mathrm{mic}} / M_{\mathrm{w}}$, where $\mathrm{Mw}$ is the weight-average molar mass of the copolymer. 
262 Values obtained for the $\mathrm{M}_{\mathrm{w} \text {,mic }}$ and $\mathrm{M}_{\mathrm{w}}$, hydrodynamic radius from dynamic light scattering are 263 listed in Table 1. Calculation of the thermodynamic radius (effective hard-sphere radius) from the 264 thermodynamic volume of the micelles and aggregation number is not strictly applicable for this 265 polymer forming non-spherical micelles. The increase in hydrodynamic radius is consistent with 266 micelle shape change, as the hydrodynamic radii of spherical micelles of block copoly(oxyalkylene)s 267 are almost independent of temperature (Booth and Attwood, 2000; Booth et al., 2006). The association 268 numbers of the ESE copolymer increase dramatically with increasing temperature, which corresponds 269 to the transition of spherical micelles to elongated micelles at higher temperatures. At $25^{\circ} \mathrm{C}$, the value 270 of $N_{\mathrm{w}}$ is 146 and, given that the specific volume of poly(oxystyrene) at $25^{\circ} \mathrm{C}$ is $0.87 \mathrm{~cm}^{3} \mathrm{~g}^{-1}$, the 271 average core volume of a micelle is approximately $200 \mathrm{~nm}^{3}$ and, if the micelles were spherical, the core 272 radius would be $3.64 \mathrm{~nm}$. Taking the length of an S unit to be $0.363 \mathrm{~nm}$ (Flory, 1969), the extended 273 length of an $S_{8}$ block is approximately $3 \mathrm{~nm}$. Hence the $S_{8}$ blocks would be over stretched in a spherical 274 core, and an elongated core would result. Considering the transition temperature is ca. $18{ }^{\circ} \mathrm{C}$, the 275 solution at $25^{\circ} \mathrm{C}$ is a mixture of spherical (compact) micelles and wormlike (elongated) micelles, 276 which is already confirmed by the evidence from DLS.

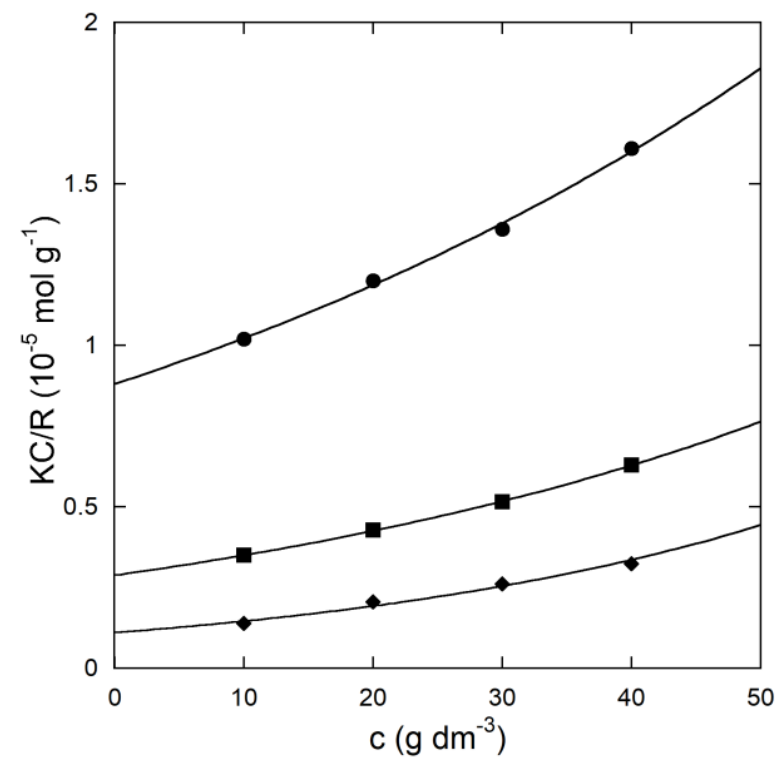


Table 1. Micelle properties of copolymer $\mathrm{E}_{16} \mathrm{~S}_{8} \mathrm{E}_{16}$ in aqueous solution ${ }^{\text {a }}$

\begin{tabular}{ccccccc}
\hline$T /{ }^{\circ} \mathrm{C}$ & $\begin{array}{c}M_{\mathrm{w}, \mathrm{mic} /} \\
10^{5} \mathrm{gmol}^{-1}\end{array}$ & $N_{\mathrm{w}}$ & $r_{\mathrm{h}}{ }^{\mathrm{b}} / \mathrm{nm}$ & $\begin{array}{c}D_{\mathrm{app}}^{\mathrm{b}} / \\
\mu \mathrm{m}^{2} \mathrm{~s}^{-1}\end{array}$ & $r_{\mathrm{h}}{ }^{\mathrm{c}} / \mathrm{nm}$ & $\begin{array}{c}D_{\mathrm{app}}{ }^{\mathrm{c}} / \\
\mu \mathrm{m}^{2} \mathrm{~s}^{-1}\end{array}$ \\
\hline 15 & 1.14 & 48 & 5.8 & & & \\
25 & 3.45 & 146 & 7.2 & 34.3 & 7.2 & 33.9 \\
40 & 9.09 & 384 & 11.2 & 31.4 & 12.6 & 27.6 \\
\hline
\end{tabular}

282

283

284

285

286

287

288

289

290

291

292

293

294

295

296

297

298

299

300

a Estimated uncertainties: \pm 1 in $r_{\mathrm{h}} ; \pm 10 \%$ in $M_{\mathrm{w}, \text { mic }}$ and $N_{\mathrm{w}}$.

b Measured by DLS

c Measured by TDA

\section{TDA}

This work is the first attempt to employ Taylor dispersion analysis to investigate the effect of temperature on the micellisation of polymeric surfactants. Compared to light scattering, TDA is less sensitive to dust particles and does not require strict sample preparation/filtration. A dilute copolymer solution above CMC was used as running buffer to prevent micellar dissociation during diffusion.

Compared to DLS that required a few millilitres of sample solution for filtration and measurement, TDA technique only utilised several hundred nanolitres for the measurement at two temperatures. Fig. 5(a) shows a standard TDA profile of a $1 \% \mathrm{w} / \mathrm{v}$ copolymer $\mathrm{E}_{16} \mathrm{~S}_{8} \mathrm{E}_{16}$ solution at $25^{\circ} \mathrm{C}$. The sample was run in triplicates. For convenience, the hydrodynamic radii and diffusion coefficients measured by TDA are also included in Table 1. The Skewed taylorgram in Fig. 5 is due to the minor interaction of micelles with the walls of the uncoated capillary. Nonetheless this deviation had no effect on the hydrodynamic radii, as the Viscosizer 200 fitting algorithm demonstrated that the data obtained had a 98\% fit to that of the sample. This indicates an appropriate Taylor dispersion profile. As discussed above, the micellar solution of ESE copolymer has a spherical-to-elongated transition temperature at ca. 
$30118{ }^{\circ} \mathrm{C}$. It is assumed that a mixture of spherical and elongated micelles co-exists in the solution at $25^{\circ} \mathrm{C}$. 302 With a further increase of temperature to $40{ }^{\circ} \mathrm{C}$, the shape transition is considered to complete, and the 303 worm-like micelles are dominant. Hence, like SLS, the size measured by TDA is an apparent value for 304 all the micelle species in the solution. As seen in Table 1 , the hydrodynamic radius at $40{ }^{\circ} \mathrm{C}$ is 305 approximately double than that at $25^{\circ} \mathrm{C}$ due to shape transition and size growth of worm-like micelles. 306 Fig. 5(b) shows the TDA profiles of $1 \% \mathrm{w} / \mathrm{v}$ copolymer solution at 25 and $40{ }^{\circ} \mathrm{C}$ under the same 307 measurement conditions. The shorter elution time at $40^{\circ} \mathrm{C}$ is attributed to lower viscosity of running 308 buffer and relatively larger micelle particles that are expected to show less radial diffusion within the 309 capillary compared to small micelles. Hence the TDA data also demonstrate the formation of elongated 310 micelles at higher temperatures. It is clearly seen in Table 1 that the results from TDA and DLS are in 311 good agreement. The values of hydrodynamic radii and diffusion coefficients obtained by these two 312 techniques are very close, which suggest that TDA is reliable alternative to DLS for size measurement.

313 However, it should be noted that TDA measures the weight- average hydrodynamic radius and

314 diffusion coefficient with a mass concentration-sensitive detector whilst DLS leads to z-average values 315 of hydrodynamic radius and diffusion coefficient. These two techniques should only report the same 316 hydrodynamic radius for monodisperse samples (Chamieh et al., 2015). 

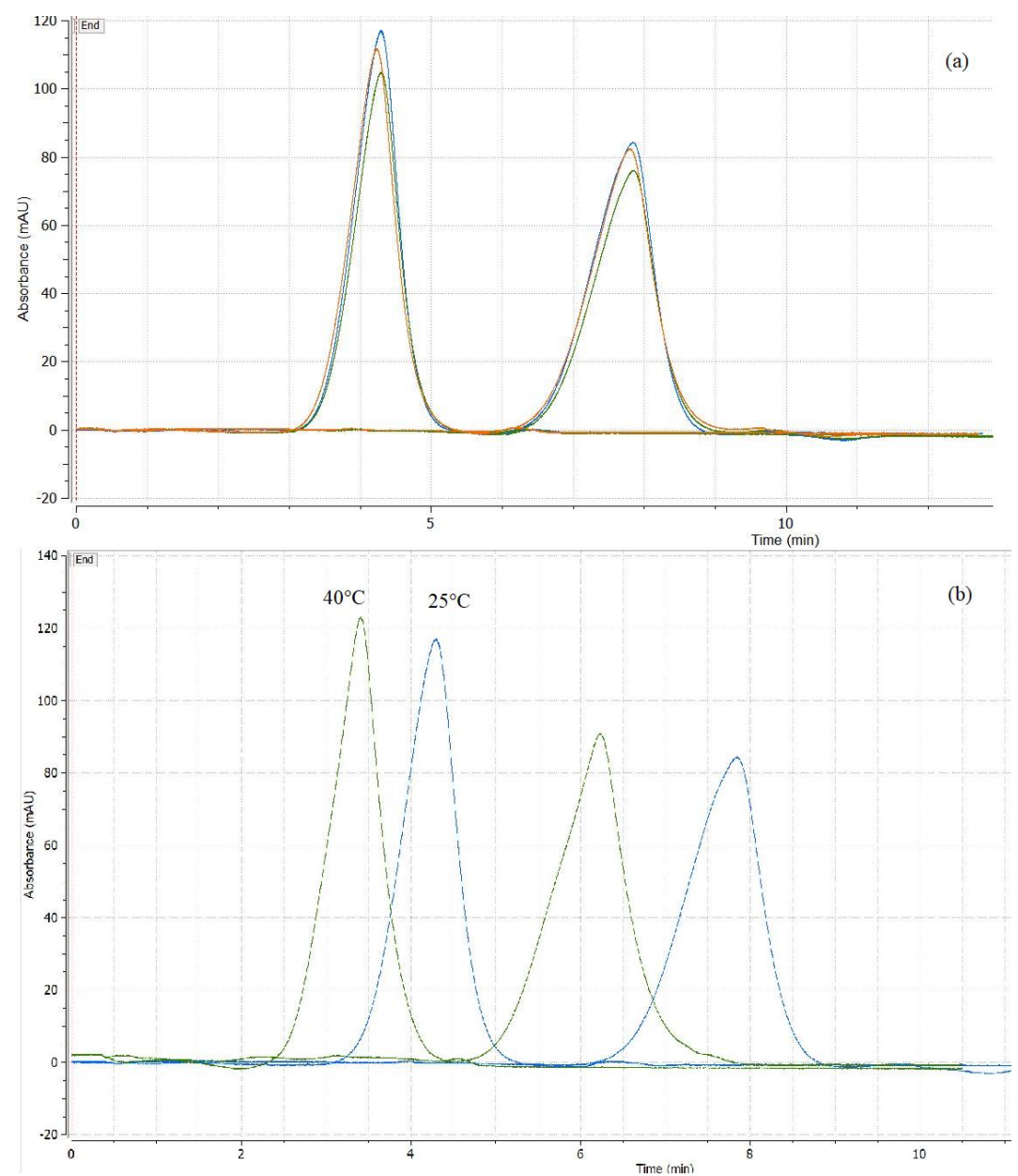

319 Figure 5. (a) TDA profile showing an overlay of three runs for a $1 \% \mathrm{w} / \mathrm{v}$ copolymer $\mathrm{E}_{16} \mathrm{~S}_{8} \mathrm{E}_{16}$ solution at $25^{\circ} \mathrm{C}$; (b) Comparison of TDA profiles of the copolymer solution at 25 and $40^{\circ} \mathrm{C}$.

\section{Drug solubilisation.}

The solubilisation of model drug, terfenadine (Fig. 6), in dilute micellar solutions of the ESE copolymer was investigated with comparison to Pluronic F127 (EPE triblock copolymer). The solubilisation capacity $\left(S_{\mathrm{cp}}\right)$ was expressed as milligram drug per gram of copolymer $\left(\mathrm{mg} \mathrm{g}^{-1}\right)$. The solubility of terfenadine in the water $\left(0.01 \mathrm{mg} \mathrm{ml}^{-1}\right.$ at $\left.30{ }^{\circ} \mathrm{C}\right)$ was subtracted from the solubility of terfenadine $(S)$ in micellar solutions to determine the amount of drug solubilised in the micelles. The solubilisation capacities for terfenadine in 1 and $2 \% \mathrm{w} / \mathrm{v}$ micellar solutions of the copolymers at 25 and $37^{\circ} \mathrm{C}$ are listed in Table 2. 


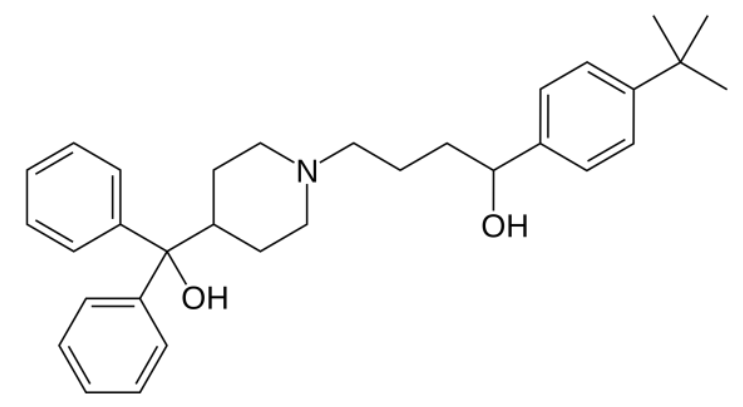

Figure 6. Molecular structure of terfenadine.

As seen in Table 2, the solubilities of terfenadine in the micellar solutions are much higher than that in water, e.g. nearly 20 -fold increase in $1 \%$ w/v ESE solution at $25^{\circ} \mathrm{C}$. The ESE copolymer shows enhanced solubilisation capacities (3-fold) than F127 under the same conditions due to the higher hydrophobicity of the core-forming $\mathrm{S}$ blocks compared to $\mathrm{P}$ blocks. It was known from our previous work that the hydrophobic core is the domain for drug solubilisation. Furthermore, elongated micelles were formed in the micellar solutions of ESE copolymer, which have larger hydrophobic core volume than spherical micelles and thus show higher solubilisation efficiency. Increasing the temperature from 25 to $37{ }^{\circ} \mathrm{C}$ leads to an enhancement of the solubilisation capacities of ESE copolymer. This is probably attributed to the complete transition to elongated micelles at $37{ }^{\circ} \mathrm{C}$ and size growth of wormlike micelles. An increase of concentration of micellar solutions shows no significant influence on the solubilisation capacities of ESE copolymer. The number of micelles increase with concentration, which leads to a higher drug solubility. However, micellar interaction at higher concentration could hinder the growth of micelles. Hence the solubilisation capacities remain the same or even a bit lower at higher concentrations. A similar tendency is also demonstrated for F127. 


\begin{tabular}{ccccc}
\hline & $T /{ }^{\circ} \mathrm{C}$ & Conc. $/ \% \mathrm{w} / \mathrm{v}$ & $S / \mathrm{mg} \mathrm{ml}^{-1}$ & $S_{\mathrm{cp}} / \mathrm{mg} \mathrm{g}^{-1}$ \\
\hline ESE & 25 & 1.0 & 0.188 & 18.8 \\
& & 2.0 & 0.337 & 16.9 \\
& 37 & 1.0 & 0.286 & 28.6 \\
& & 2.0 & 0.508 & 25.4 \\
F127 & 25 & 1.0 & 0.059 & 5.9 \\
& & 2.0 & 0.116 & 5.8 \\
\hline
\end{tabular}

\section{Conclusions}

a. Estimated error $\pm 10 \%$.

The synthesis and characterisation of triblock copolymer $\mathrm{E}_{16} \mathrm{~S}_{8} \mathrm{E}_{16}$ are reported. Light scattering studies indicated that, with carefully-chosen block lengths and composition, the triblock copolymer is able to self-associate in aqueous solution and form worm-like micelles at ambient temperatures. Like other diblock copo(oxyalkylene)s of this kind, a transition temperature from spherical to elongated micelles was observed for $\mathrm{E}_{16} \mathrm{~S}_{8} \mathrm{E}_{16}$ at ca. $18{ }^{\circ} \mathrm{C}$. The shape transition was also demonstrated by TDA measurement, which shows a near doubling of micellar size at $40{ }^{\circ} \mathrm{C}$. The results from light scattering and TDA are in good agreement on hydrodynamic radii and diffusion coefficients at the temperatures investigated. The sample sparing capability of TDA provides for its more extensive use in this field. The ESE copolymer shows much greater solubilisation capacities for a poorly water-soluble model drug than a Pluronic comparator because of the hydrophobic nature of $\mathrm{S}$ blocks and formation of worm-like micelles for the ESE copolymer. 
370 We thank Dr. Zhuo Yang for advice with copolymer synthesis and characterisation.

371

372 References

373

374 Adams M. L., Lavasanifar A., Kwon G. S., 2003. Amphiphilic block copolymers for drug delivery. J.

375 Pharm. Sci., 92, 1343-1355.

376

377 Aris R., 1956. On the dispersion of a solute in a fluid flowing through a tube. Proc. R. Soc. A, 235, $378 \quad 67-77$.

379

380

Attwood D., Zhou Z., Booth C., 2007. Poly(ethylene oxide) based copolymers: solubilisation capacity 381 and gelation. Expert Opin. Drug Deliv., 4, 533-546.

382

Booth C., Attwood D., 2000. Effects of block architecture and composition on the association 384 properties of poly(oxyalkylene) copolymers in aqueous solution. Macromol. Rapid Commun., 21, $385 \quad 501-527$.

386

Booth C., Attwood D., Price C., 2006. Self-association of block copoly(oxyalkylene)s in aqueous 388 solution. Effects of composition, block length and block architecture. Phys. Chem. Chem. Phys., 8, 389 $3612-3622$.

390

391 Carnahan N. F., Starling K. E., 1969. Equation of State for Nonattracting Rigid Spheres. J. Chem. 392 Phys., 51, 635-636. 
394 Chaibundit C., Ricardo N. M. P. S., Crothers M., Booth C., 2002. Micellization of 395 diblock(oxyethylene/oxybutylene) copolymer $\mathrm{E}_{11} \mathrm{~B}_{8}$ in aqueous solution. Micelle size and shape. Drug 396 solubilization. Langmuir, 18, 4277-4283.

397

398 Chaibundit C., Sumanatrakool P., Chinchew S., Kanatharana P., Tattershall C. E., Booth C., Yuan X. 399 F., 2005. Association properties of diblock copolymer of ethylene oxide and 1,2-butylene oxide: $E_{17} B_{12}$ 400 in aqueous solution. J. Colloid Interface Sci., 283, 544-554.

401

402 403

Chamieh J., Davanier F., Jannin V., Demarne F., Cottet H., 2015. Size characterization of commercial micelles and microemulsions by Taylor dispersion analysis. Int. J. Pharm., 492, 46-54.

404

405

Chiappetta D. A., Sosnik A., 2007. Poly(ethylene oxide)-poly(propylene oxide)-poly(ethylene oxide) 406 block copolymer micelles as drug delivery agents. Improved hydrosolubility, stability and 407 bioavailability of drugs. Eur. J. Pharm. Biopharm., 66, 303-317.

408

Cottet H., Martin M., Papillaud A., Souaïd E., Collet H., Commeyras A., 2007. Determination of 410 dendrigraft poly-L-lysine diffusion coefficients by Taylor dispersion analysis. Biomacromolecules, 8 , $4113235-3243$.

412

413 Crothers M., Zhou Z., Ricardo N. M. P. S., Yang Z., Taboada P., Chaibundit C., Attwood D., Booth C., 414 Solubilisation in aqueous micellar solutions of block copoly(oxyalkylene)s. Int. J. Pharm., 293, 91-100. 415

416 Flory P. J., 1969. Statistical mechanics of chain molecules. Interscience, New York, p. 165. 
418 Grindel J. M., Jaworski T., Piraner O., Emanuele R. M., Balasubramanian M., 2002. Distribution, 419 metabolism, and excretion of a novel surface-active agent, purified poloxamer 188, in rats, dogs, and 420 humans. J. Pharm. Sci., 91, 1936-1947.

421

422 Hawe A., Hulse W. L., Jiskoot W., Forbes R. T., 2011. Taylor dispersion analysis compared to 423 dynamic light scattering for the size analysis of therapeutic peptides and proteins and their aggregates. 424 Pharm. Res., 28, 2302-2310.

425

426 Heatley F., Yu G. E., Draper M. D., Booth C., 1991. Analysis of the ${ }^{13}$ C-NMR spectra of poly(styrene 427 oxide) and of block and statistical copolymers of styrene oxide and ethylene oxide. Eur. Polym. J., 27, $428 \quad 471-478$.

429

Lemal P., Balog S., Geers C., Taladriz-Blanco P., Palumbo A., Hirt A. M., Rothen-Rutishauser B., 431 Petri-Fink A., 2019. Heating behavior of magnetic iron oxide nanoparticles at clinically relevant 432 concentration. J. Magn. Magn. Mater., 474, 637-642.

Taylor G., 1953. Dispersion of soluble matter in solvent flowing slowly through a tube. Proc. R. Soc. A, $435219,186-203$.

436

437 Torchilin V. P., 2001. Structure and design of polymeric surfactant-based drug delivery systems. J. 438 Control. Rel., 73, 137-172. 
440 Yang Z., Booth C., Crothers M., Attwood D., Collett J. H., Ricardo N. M. P. S., 2003. Association 441 properties of ethylene oxide/styrene oxide diblock copolymer $\mathrm{E}_{17} \mathrm{~S}_{8}$ in aqueous solution. J. Colloid 442 Interface Sci., 263, 312-317.

443

444 Yang Z., Crothers M., Ricardo N. M. P. S., Chaibundit C., Taboada P., Mosquera V., Kelarakis A., 445 Havredaki V., L. Martini G. A., Valder C., Collett J. H., Attwood D., Heatley F., Booth C., 2003 b. 446 Micellization and gelation of triblock copolymers of ethylene oxide and styrene oxide in aqueous 447 solution. Langmuir, 19, 943-950.

448

449 Zaman H., Bright A. G., Adams K., Goodall D. M., Forbes R. T., 2017. Characterisation of aggregates 450 of cyclodextrin-drug complexes using Taylor Dispersion Analysis. Int. J. Pharm., 522, 98-109.

452 Zhou Z., Chaibundit C., D’Emanuele A., Lennon K., Attwood D., Booth C., 2008. Solubilisation of 453 drugs in worm-like micelles of block copolymers of ethylene oxide and 1,2-butylene oxide in aqueous 454 solution. Int. J. Pharm., 354, 82-87.

455

456 Zhou, Z., D'Emanuele, A., Lennon, K., Attwood, D., 2009. Synthesis and micellization of linear457 dendritic copolymers and their solubilization ability for poorly water-soluble drugs. Macromolecules $458 \quad 42,7936-7944$

459 